\title{
Les protéines du cytosquelette d'actine : bien placées pour la motilité
}

\author{
Roy M. Golsteyn \\ Monique Arpin \\ Évelyne Friederich \\ Daniel Louvard
}

R.M. Golsteyn, M. Arpin, E. Friederich, D. Louvard: Institut Curie, Cnrs UMR 144 Morphogenèse et signalisation cellulaires, 26, rue d'Ulm, 75248 Paris Cedex 05, France.
Les cellules d'un organisme sont animées par une très grande variété de mouvements, qui nécessitent la coordination d'un réseau complexe de protéines formant le cytosquelette d'actine. Au centre de ce réseau se trouve I'actine, qui possède des propriétés dynamiques intrinsèques, grâce à sa capacité de former un long polymère à partir de monomères. Dans cette revue, nous discuterons trois systèmes différents qui règlent I'organisation du cytosquelette d'actine. Deux d'entre eux sont importants pour le contrôle de la polymérisation de l'actine dans des structures cellulaires différentes : I'un dépend du complexe Arp2/3, I'autre de la zyxine. Le troisième est représenté par la protéine ezrine qui, par son interaction avec les protéines membranaires et les filaments d'actine, peut régler I'organisation du cortex cellulaire en affectant la motilité et la survie.

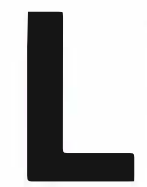

'observation des cellules vivantes permet d'apprécier la dynamique du cytosquelette d'actine. En effet, les cellules se déplacent dans plusieurs directions, émettent des projections puis les rétractent, s'applatissent et parfois s'arrondissent et se séparent pour donner deux cellules filles. Tous ces mouvements nécessitent l'intervention du cytosquelette d'actine. A l'intérieur de la cellule, ce cytosquelette peut être visualisé grâce à la phalloidine (couplée à la rhodamine), une petite molécule qui se fixe sur les filaments $d^{\prime}$ actine (ou actine-F) (figure 1). Deux types de filaments d'actine sont apparents: l'actine-F forme un réseau dense sous la membrane plasmique, mais elle est aussi présente sous la forme de câbles très structurés appelés câbles de stress, qui traversent le cytoplasme et s'ancrent à la membrane plasmique dans des structures spécialisées, les points focaux. La formation de ces différentes structures d'actine-F doit être coordonnée par des mécanismes de régulation qui contrôlent le mouvement, la différenciation, la division et la survie des cellules, donc par des événements qui dépendent du cytosquelette d'actine. La polymérisation de l'actine et les différents processus cellulaires qu'elle contrôle sont réglés de manière très fine dans le temps et dans l'espace, puisque les différentes structures d'actine sont très dynamiques dans certaines régions de la cellule, mais pas dans d'autres. Contrairement à la complexité du cytosquelette observé in vivo, les propriétés biochimiques de l'actine pure en solution sont simples. L'actine monomérique (actine-G) polymérise pour former des filaments, et cette polymérisation est contrôlée par la concentration en actine de deux façons différentes. Tout d'abord, les cinétiques de polymérisation sont différentes aux deux extrémités du filament d'actine. L'une des extrémités, appelée extrémité «barbue» ou (+), fixe des monomères d'actine à une concentration plus basse que l'autre, appelée l'extrémité «pointue» ou (-). Cette asymétrie confère une dyna- 


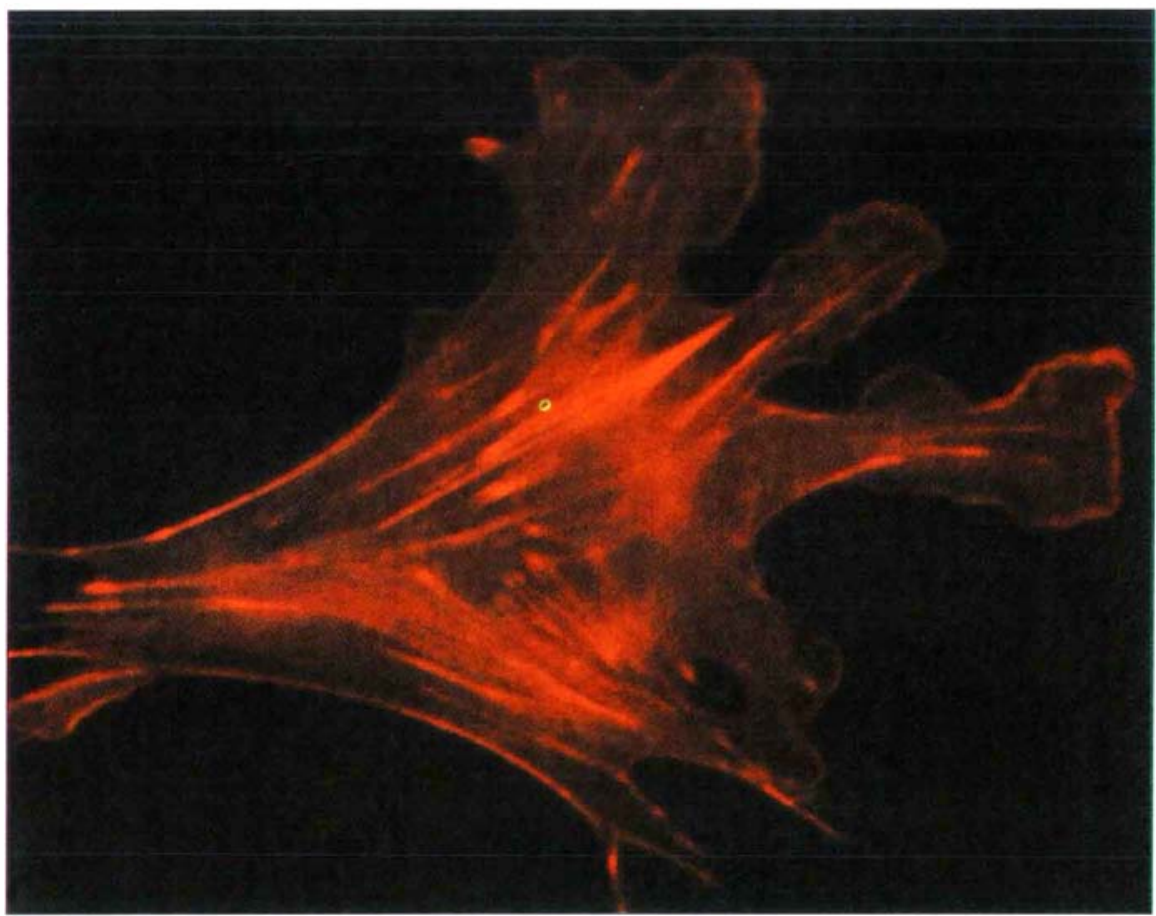

Figure 1. Fibroblaste de souris fixé, marqué avec une drogue fluorescente se fixant sur l'actine-F. Les structures les plus importantes d'actine-F sont observées dans le front de migration (correspondant aux projections sur le côté droit des cellules) et dans les faisceaux épais $d^{\prime}$ actine- $F$ qui traversent la cellule. Ces différentes structures sont produites par différents types de protéines, particulièrement le complexe Arp2/3 au front de migration, et les protéines de la famille de la zyxine présentes dans les points focaux.

mique aux filaments d'actine car elle permet à un même filament de s'allonger à l'extrémité $(+)$ et de se raccourcir à l'extrémité $(-)$, cela pour une concentration donnée d'actine. Les cellules utilisent ces propriétés pour créer de nouveaux filaments par différentes méthodes: soit la polymérisation se fait à partir de filaments préexistants par addition de monomères aux extrémités $(+)$ (ces extrémités doivent être décoiffées dans la cellule), soit de nouvelles extrémités de filaments sont créées par coupure d'un filament (processus de fragmentation). Enfin, une polymérisation de filaments de novo ou une nucléation à partir de deux ou trois monomères $d^{\prime}$ actine peuvent exister. La polymérisation d'actine est aussi modulée par des protéines collectivement appelées "protéines de liaison à l'actine" qui interagissent avec I'actine-G et/ou I'actine-F. L'activité de ces protéines est réglée par différentes voies de signalisation, par phosphorylation, par de petites molécules $\left(\mathrm{Ca}^{2+}\right.$, phospho-inositides...) ou par leur concen- ron $2 \mu \mathrm{m}$, d'où sont exclus les organites, contient de l'actine-F qui, observée en microscopie électronique, apparaît organisée en réseaux formés de courts filaments ramifiés. Dans cette région de la cellule, I'actine-F incorpore des monomères d'actine plus rapidement que dans d'autres régions de la cellule pour former de nouveaux filaments. Des études réalisées grâce à des monomères d'actine marqués avec des composés fluorescents indiquent que les nouveaux filaments se forment près de la membrane plasmique [1]. La polymérisation de l'actine crée une force suffisante pour faire avancer la membrane plasmique et, avec d'autres régions de la cellule, promouvoir la motilité cellulaire. Les filaments anciennement formés qui se trouvent à l'arrière du front de migration se dépolymérisent, relarguent des monomères d'actine qui seront réincorporés dans de nouveaux filaments près de la membrane plasmique. Ce phénomène est strictement contrôlé et contribue à la production de forces qui permettent la motilité cellulaire.

En 1999, les mécanismes mis en jeu dans la polymérisation de l'actine des lamellipodes ont pu être mieux compris, grâce à la convergence des études de reconstitution du mouvement, utilisant des bactéries et des extraits d'œufs de xénopes [2]. Ces études ont montré qu'un élément clé dans la production d'actine- $F$ est le complexe Arp2/3 (pour actin related proteins). Ce complexe constitué de 7 protéines a été à l'origine isolé à partir d'un organisme unicellulaire, Acanthamoeba castellanii, et fut identifié plus tard dans toutes les espèces eucaryotes depuis la levure jusqu'à I'homme. Le complexe Arp2/3 humain est composé de 7 protéines: Arp2 et Arp3, p41-arc, p34-arc, p21arc, p20-arc et p16-arc [3]. II est localisé dans le front de migration des cellules, qui est la région où l'on observe le taux le plus élevé de polymérisation de l'actine. Même chez la levure, dont la périphérie de la cellule est limitée par une paroi, le complexe Arp2/3 est associé avec des structures corticales d'actine qui sont les régions les plus dynamiques.

La première information sur le rôle essentiel du complexe Arp2/3 dans la polymérisation de l'actine a été obte- 
nue par des études réalisées avec la bactérie Listeria monocytogenes [4] (figure 2). Les Listeria sont des bactéries à Gram positif, parfois présentes dans les produits laitiers non pasteurisés. Après ingestion, les bactéries pénètrent et se déplacent dans les cellules jusqu'à ce qu'elles atteignent la membrane plasmique, et soient englouties par la cellule adjacente [5]. L'intérêt du modèle de Listeria dans l'étude de la polymérisation de l'actine a été reconnu pour la première fois par Lew Tilney et Dan Portnoy en 1989 [6]. En examinant par microscopie électronique des macrophages infectés par des Listeria, ces auteurs ont observé la présence de "comètes" d'actine derrière les bactéries. L'organisation de l'actine-F dans les comètes et dans le front de migration des cellules en mouvement est similaire; on observe beaucoup de petits filaments en réseaux, avec leurs extrémités $(+)$ proches de la bactérie (figure 3). Lorsque les cellules infectées par Listeria sont traitées par la toxine cytochalasine $D$, qui se fixe à l'extrémité (+) des filaments d'actine et bloque la polymérisation, les bactéries ne bougent plus. De plus, I'utilisation d'actine fluorescente indique que les monomères d'actine s'accumulent préférentiellement à l'extrémité des filaments proche de la bactérie, indiquant ainsi que le mouvement des Listeria est produit par la polymérisation de l'actine à l'extrémité $(+)$, tout comme dans le front de migration. L'identité des mécanismes biochimiques impliqués dans le mouvement des Listeria et dans celui des cellules eucaryotes a été confirmée par l'isolement de protéines extraites de plaquettes, capables de restaurer le mouvement des bactéries. Ainsi, le mouvement des Listeria a été reconstitué dans un système défini avec des protéines purifiées parmi lesquelles le complexe Arp2/3 est un composant essentiel [7].

L'analyse biochimique et cinétique de I'interaction du complexe Arp2/3 avec I'actine indique que le complexe se fixe à l'extrémité (-) des filaments $d^{\prime}$ actine et pourrait stabiliser les dimères et les trimères $d^{\prime}$ actine nécessaires pour déclencher de nouvelles polymérisations [8]. Cela suggère que le complexe Arp2/3 possède une activité de "nucléation », c'est-àdire la capacité d'assembler des monomères $d^{\prime}$ actine, à partir desquels

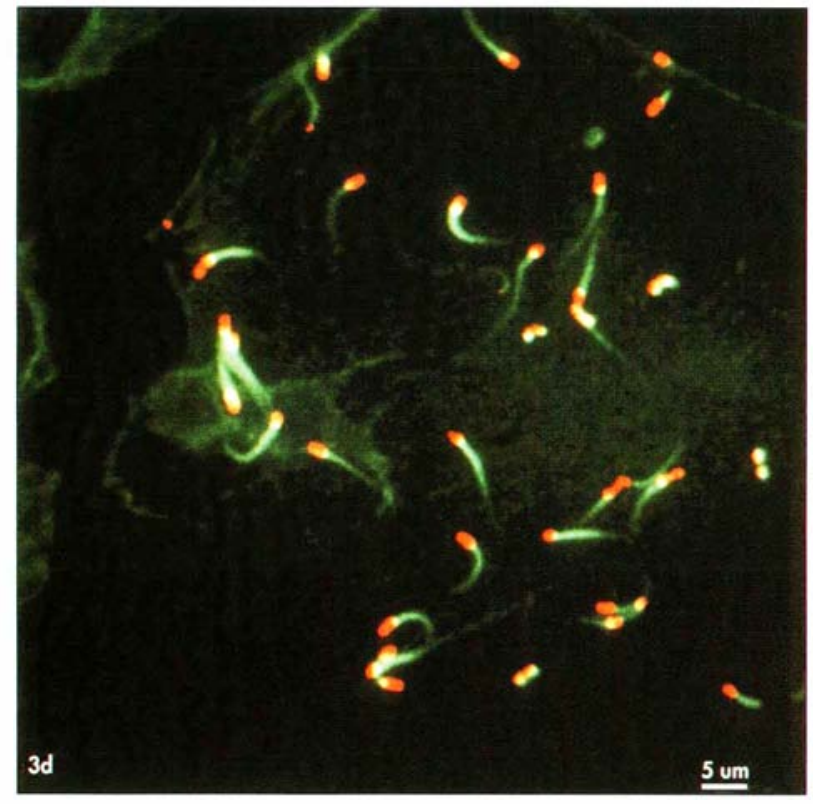

Figure 2. Cellules en culture infectées par la bactérie Listeria monocytogenes (en rouge). Ces bactéries expriment, à leur surface, la protéine ActA qui leur permet de recruter des protéines du cytosquelette de la cellule hôte. La polymérisation de l'actine par les protéines de la cellule hôte entraîne la formation d'une comète $d$ 'actine- $F$ (en vert) capable de promouvoir le mouvement de la bactérie. Ce type de mouvement est en fait une version simplifiée de phénomènes plus

complexes observés lors du mouvement des cellules. L'analyse moléculaire du mouvement des Listeria, réalisée par différents laboratoires, a été déterminante pour l'élucidation du rôle du complexe Arp2/3 et zyxine dans la polymérisation de l'actine dans les cellules humaines. (Photo produite par le Pr P. Cossart, Institut Pasteur, Paris, France.)

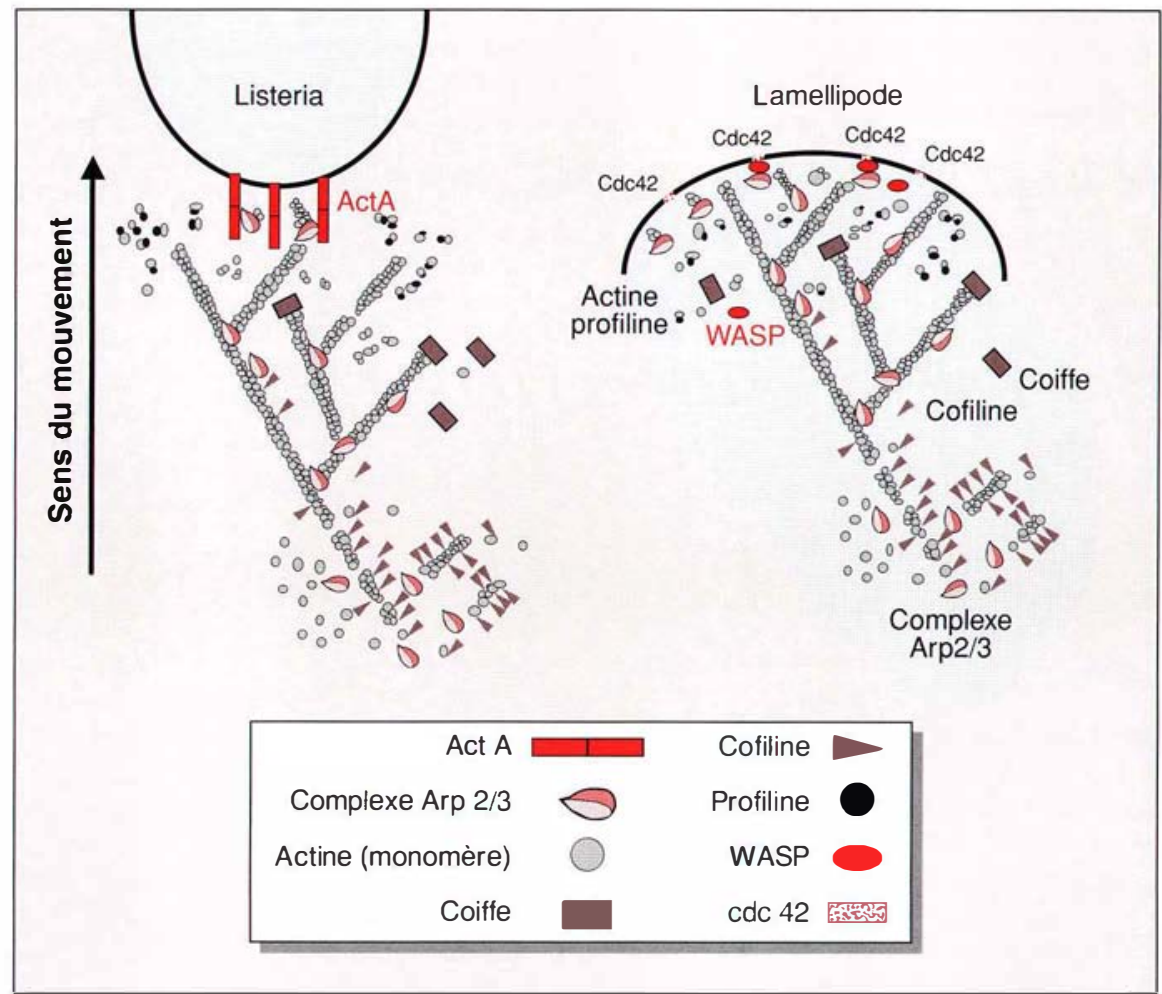

Figure 3. Organisation similaire de l'actine dans la comète de Listeria et dans le lamellipode d'une cellule en migration. La protéine bactérienne ActA est responsable de la formation de la comète d'actine, alors que, dans le lamellipode, des protéines de la famille WASP ont une activité très similaire à celle de la protéine ActA. (Schéma réalisé par Julie Fradelizi, Institut Curie, Paris, France.) 


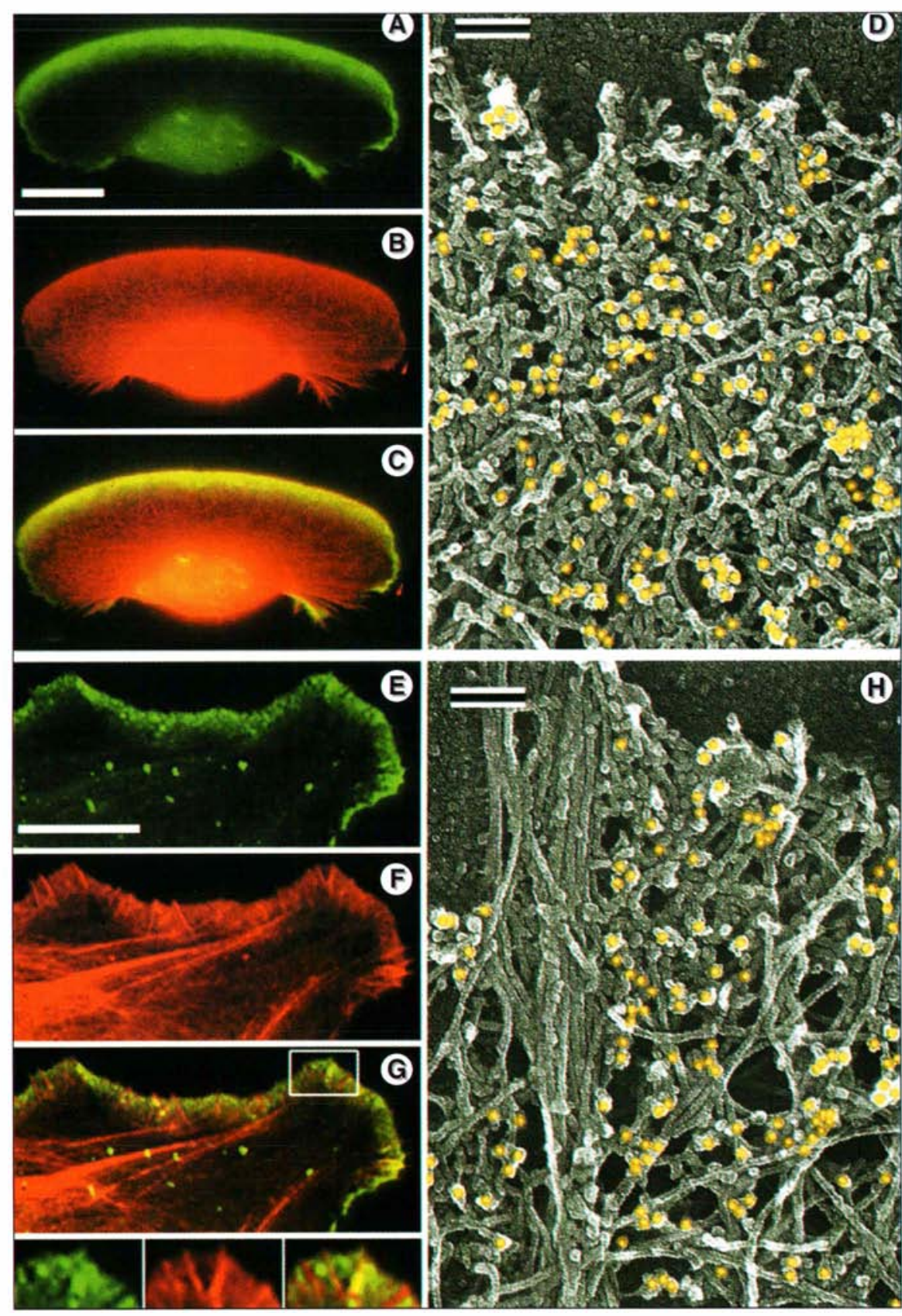

Figure 4. Localisation du complexe Arp2/3 dans les lamellipodes (a-c et e-g). Les cellules sont des kératinocytes (a-c) ou des fibroblastes de xénope (e-g). $L^{\prime}$ 'actine est en rouge et le complexe Arp2/3 en vert. (D) et $(\boldsymbol{H})$ représentent la visualisation en microscopie électronique des complexes Arp2/3 (jaune) dans un kératocyte (D) ou un fibroblaste (H). (Photo reproduite avec la permission de G.G. Borisy du site web: http://borisy.bocklabs.wisc.edu.)

de nouveaux filaments seront formés (in vitro, il s'agit d'une étape limitante). Cependant, le système présente plusieurs contraintes: il doit être activé par une autre protéine, son activité est maximale lorsqu'il est fixé sur le côté d'un filament préexistant, et il reste attaché aux filaments dont il a induit spectaculaires, Svitkina et Borisy ont montré que le complexe Arp2/3 est présent là où de nouveaux filaments se sont formés [9] (figure 4). De plus, ces branchements forment un angle de $70^{\circ}$ définissant un réseau orthogonal, qui ressemble à ceux décrits dans le front de migration des cellules et dans la comète de Listeria.

En dépit de ces propriétés très intéressantes, le complexe Arp2/3 n'a qu'une très faible activité de "nucléation " lorsqu'il est ajouté à des solutions d'actine-G, ce qui suggère que d'autres composants sont nécessaires. Parmi ceux-ci, un candidat a été proposé: il s'agit $\operatorname{de} A c t A$, une protéine bactérienne présente à la surface des Listeria. L'addition de ActA à une solution d'actine contenant le complexe Arp2/3 augmente considérablement le taux de polymérisation de I'actine-F par rapport au taux observé en I'absence du complexe Arp2/3 ou de ActA. Les cellules eucaryotes ne contenant normalement pas ActA, il a été proposé qu'une autre protéine, coopérant avec le complexe Arp2/3 devait exister, afin de stimuler la polymérisation de l'actine. En effet, les travaux de Roghatgi et al. ont démontré que les protéines de la famille WASP activent également le complexe Arp2/3 [10].

L'identification de WASP et des protéines apparentées a permis d'établir un lien entre certaines maladies et la polymérisation de l'actine dans le front de migration. WASP est le produit du gène WAS, qui existe sous forme mutée chez les patients souffrant du syndrome de WiskottAldrich. Ce syndrome, qui affecte quatre enfants mâles sur un million, est lié au chromosome $X$ et se caractérise par de sévères thrombocytopénies, de l'eczéma et un déficit immunitaire $\left(m / s\right.$ 1998, $n^{\circ} 11, p .1280$; $\mathrm{m} / \mathrm{s}$ 1996, n० 10, p. 1173).

Le rôle de WASP dans l'organisation des filaments d'actine a d'abord été suggéré lorsque son association avec cdc42, une petite GTPase ayant un rôle régulateur dans la polymérisation de l'actine, a été mise en évidence [11] (m/s 1996, $\left.n^{\circ} 12, p .1421-3\right)$. L'analyse de la structure primaire de WASP a révélé que, outre le site de liaison aux petites GTPases, cette protéine contient d'autres domaines d'interaction tels qu'un domaine WH1 présent également parmi les 
membres de la famille VASP/ena, un domaine riche en proline qui permet la liaison aux domaines SH3. Dans la partie carboxy-terminale, un domaine WH2/VCA contient de courtes séquences $d$ 'acides aminés trouvés dans la verproline (V) et la cofiline (C) deux protéines qui interagissent avec I'actine-G et I'actine- $F$, ainsi qu'une séquence nécessaire à la liaison du complexe Arp2/3.

Tout comme le complexe Arp2/3, la famille de protéines WASP est conservée depuis la levure jusqu'à I'homme, et contient au moins trois sous-familles: VASP/ena, les protéines Scar, et WASP. La confirmation du rôle de WASP dans la production de forces créées par la polymérisation d'actine a été apportée par une expérience utilisant des microbilles recouvertes de la protéine WASP. Lorsque ces billes sont placées dans un extrait cellulaire, elles recrutent de l'actine, forment des comètes, se déplacent et ce mouvement est dépendant du complexe Arp2/3 [12].

L'identification du complexe Arp2/3 et de WASP a enfin permis d'expliquer les mécanismes de polymérisation de I'actine cellulaire. Ces études ont montré que ces mécanismes peuvent être plus divers que ce qui avait été anticipé, tout en étant prévisibles par simple observation de la cellule.

\section{Juste derrière - La zyxine}

En dehors du front de migration, les points focaux représentent d'autres structures riches en actine-F. Ces points d'ancrage à la matrice extracellulaire n'ont pas seulement une fonction mécanique mais sont aussi de véritables "centres" de signalisation. Tout comme dans le front de migration, ce sont les propriétés dynamiques de l'actine associée aux points focaux qui jouent un rôle essentiel lors de la migration cellulaire. En revanche, dans les points focaux, I'organisation de I'actine-F est très différente de celle observée dans le front de migration. Contrairement à ceux des lamellipodes, les microfilaments ne sont pas organisés en réseau orthogonal, mais en faisceaux qui sont attachés à la membrane plasmique. Les extrémités (+) des microfilaments sont orientées vers la membrane plasmique et sont en étroit contact avec un complexe protéique cytoplasmique associé aux intégrines, ces récepteurs transmembranaires qui ancrent la cellule à la matrice extracellulaire. L'organisation de l'actine-F des points focaux étant très différente de celle du front de migration, il n'est peut être pas surprenant qu'ils ne contiennent pas le complexe Arp2/3, et qu'un autre mode de polymérisation de l'actine existe dans ces structures.

II est bien établi que I'activation des intégrines, de concert avec des signaux provenant des récepteurs tyrosine kinase, déclenche la cascade des évenements qui mènent à la formation d'un complexe protéique ancré aux microfilaments. Ces voies de signalisation activent les petites GTPases de la famille de rho, de véritables «interrupteurs" moléculaires qui jouent un rôle primordial dans le contrôle de la dynamique et de I'organisation du cytosquelette [13]. Ainsi, une des fonctions de Rho est d'activer la myosine \| en contrôlant l'état de phosphorylation de la sousunité régulatrice de la myosine II [14]. Sous sa forme activée, la myosine II forme des dimères bipolaires qui s'associent à deux filaments d'actine, favorisant ainsi leur organisation en faisceaux [15]. Si l'importance de la myosine II dans I'assemblage des câbles de stress est indéniable, le mécanisme par lequel l'actine polymérisée est formée au niveau des points focaux demeure mal compris. L'étude de l'assemblage des câbles de stress par vidéomicroscopie suggère l'existence d'un précurseur cytoplasmique comportant de I'actine polymérisée [16]. De même, I'injection de la petite GTPase rho dans des cellules en culture induit la formation de câbles de stress dans le cytoplasme, en l'absence de complexes d'adhérence [17l. Des faisceaux d'actine pourraient donc être assemblés dans le cytoplasme, avant d'être ancrés aux intégrines par l'intermédiaire de protéines telles que la taline ou l' $\alpha$-actinine, qui interagissent directement avec la partie cytoplasmique des intégrines. Cependant, des données expérimentales suggèrent également que des filaments d'actine sont formés de novo aux points focaux. En effet, I'actine monomérique est rapidement incorporée au niveau de ces structures dans des modèles de cellules perméabilisées, suggérant la présence de sites de nucléation de la polymérisation de l'actine.

Si le complexe Arp2/3 est absent des points focaux, il est important de noter la présence de la zyxine, initialement découverte par Mary Beckerle, à l'Université d'Utah (USA) [18]. De façon comparable à l'identification de WASP, c'est de nouveau le modèle de Listeria qui a prouvé la fonction de cette protéine dans le contrôle de la polymérisation de I'actine. La zyxine est la première protéine de mammifère pour laquelle des propriétés structurales et fonctionnelles communes avec ActA de Listeria ont été mises en évidence [19]. La zyxine appartient à une famille de protéines localisées dans les lamellipodes, les jonctions adhérentes et dans les points focaux. Elle interagit avec des protéines du cytosquelette, I' $\alpha$-actinine, les protéines VASP/Mena, ainsi qu'avec Vav, un facteur d'échange des petites GTPase $\operatorname{Rac}\left(m / s 1995, n^{\circ} 7, p .1045\right)$ et de Rho (m/s 1996, $\left.n^{\circ} 11, p .1235-40\right)$. Sa partie carboxy-terminale, riche en résidus cystéine et histidine comporte trois domaines LIM. Ces domaines forment des structures appelées "doigts de zinc " qui sont impliquées dans les interactions protéineprotéine.

Les propriétés biochimiques et biologiques de la zyxine suggèrent qu'elle est impliquée dans le contrôle de la dynamique de l'actine. D'une part, le ciblage de cette protéine à la face interne de la membrane plasmique grâce à une séquence CAAX, induit la formation d'extensions membranaires riches en actine; d'autre part, la partie amino-terminale de la zyxine est riche en proline et comporte des motifs "FPPPP » (consensus: E/DF PPPPXD/E). Ces motifs, initialement identifiés et caractérisés dans ActA de Listeria monocytogenes [20], sont également présents dans la LPP, un nouveau membre de la famille de la zyxine 121| ainsi que dans la vinculine. Ces propriétés de la zyxine suggèrent qu'elle pourrait avoir un rôle dans I'assemblage de complexes multiprotéiques participant à la régulation de la dynamique de l'actine. Ainsi, une des fonctions de la zyxine serait de servir de "point d'amarrage" des protéines de la famille de VASP/Mena (figure 5). 


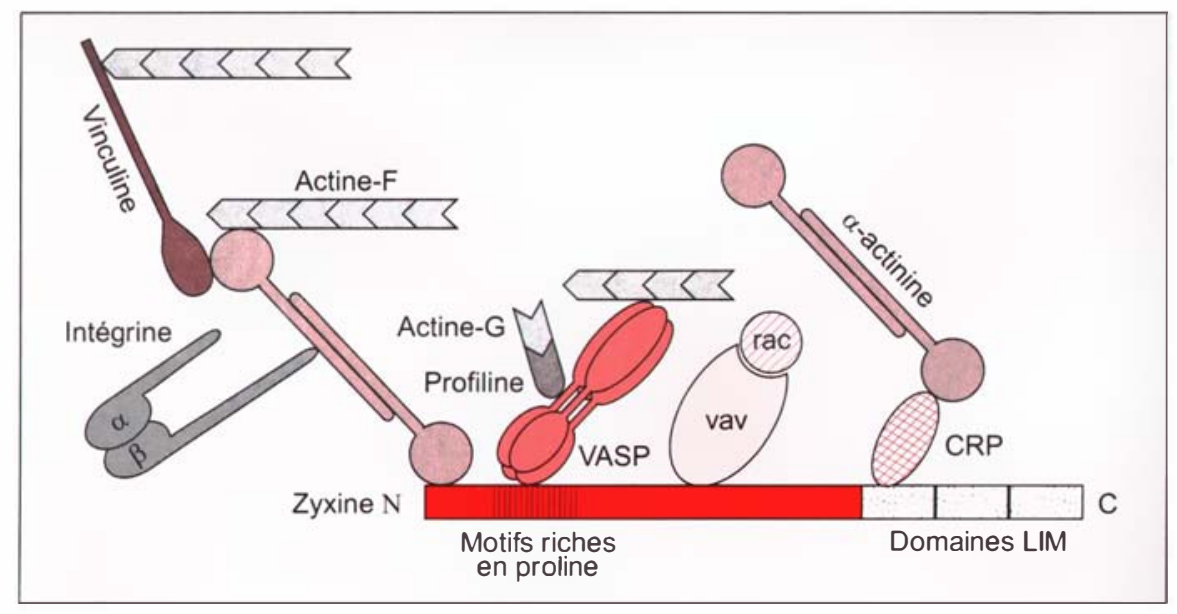

Figure 5. Représentation schématique des caractéristiques structurales de la zyxine et des protéines apparentées. La zyxine contient deux domaines: un domaine riche en proline et un domaine LIM. IIs contiennent tous deux de nombreux sites $d^{\prime}$ interaction avec $d^{\prime}$ autres protéines, dont certaines interagissent avec le cytosquelette d'actine. Nous décrivons ici l'interaction avec VASP, mais d'autres partenaires tels que CRP (cysteine-rich protein), VAV, I' $\alpha$-actinine interagissent aussi avec la zyxine. La liste complète des protéines liant la zyxine n'est pas connue. Néanmoins, il est clair que la zyxine et les protéines apparentées ont un rôle de régulation du cytosquelette d'actine. (Schéma réalisé par Julie Fradelizi, Institut Curie, Paris, France.)

Les motifs «FPPPP» sont des unités modulaires capables de lier les membres de la famille VASP/Mena, dont on pense qu'ils jouent un rôle essentiel dans le contrôle de la dynamique du cytosquelette. VASP a été d'abord décrit comme un substrat de kinases dépendantes de l'AMPc et du GMPc, activées lors de l'inhibition de la réaction d'agrégation des plaquettes sanguines. La protéine Mena, un autre membre de cette famille, est détectée exclusivement dans le cerveau [22]. Ces protéines sont apparentées à WASP et contiennent comme celle-ci un domaine EVH1 ainsi que des séquences riches en proline qui sont importantes pour I'interaction avec la profiline [23].

Depuis la détection de la protéine VASP à la surface de Listeria monocytogenes, sa participation au contrôle de la polymérisation de l'actine a été proposée [24]. La vitesse du mouvement de bactéries qui produisent des mutants d'ActA, tronqués des séquences riches en proline, est réduite de trois à six fois. Des expériences de reconstitution in vitro du mouvement de Listeria monocytogenes ont récemment confirmé un saire à la polymérisation de l'actine sont étroitement liés. Ainsi, la zyxine ou d'autres protéines comportant des motifs "FPPPP " seraient capables de moduler l'activité des protéines VASP/Mena en recrutant ces protéines à des sites dynamiques de la cellule. L'observation que la LPP, apparentée à la zyxine, recrute VASP lorsqu'elle est ciblée à une localisation ectopique, est en faveur d'un tel mécanisme [21]. De plus, des peptides comportant le motif consensus d'interaction avec VASP/Mena sont capables de déplacer VASP hors des points focaux lorsqu'ils sont introduits dans des cellules en culture [20]. S'il est donc probable que le recrutement de VASP/Mena au niveau des points d'adhérence à la matrice extracellulaire dépende majoritairement des protéines comportant des motifs "FPPPP», il n'est pas exclu que VASP/Mena soit également directement recrutée aux sites d'incorporation de l'actine à la face interne de la membrane plasmique [26].

\section{Et au-dessus - L'ezrine}

Nous avons décrit les deux types de structure d'actine, visibles dans la cellule, comme deux entités indépendantes, mais en réalité elles ne le sont pas. Ainsi, lorsqu'une cellule se déplace et crée un front de migration, elle doit établir de nouveaux points de contact. Cela requiert un niveau supérieur d'organisation tout comme "la décision" d'une cellule de se différencier, de se diviser ou de se déplacer de nouveau, voire de mourir. Tous ces processus nécessitent le cytosquelette d'actine et vont dépendre de l'action coordonnée de la polymérisation/dépolymérisation des filaments d'actine, mais aussi de leur association avec la membrane plasmique. Le mode d'interaction des filaments d'actine avec la membrane peut être très varié, mais nous limiterons cette discussion à une famille de protéines, les ERM (ezrine, radixine, moésine). L'ezrine a été isolée en 1983 par A. Bretscher de I'Université Cornell, à New York (USA) (d'où son nom en hommage à son fondateur, Ezra Cornell) comme un composant des microvillosités des cellules intestinales [27], tandis que la moésine et la radixine ont été caractérisées plus tard par des approches de biologie 
moléculaire. Les ERM présentent un fort degré d'homologie (75\% d'identité). En outre, leur domaine aminoterminal est conservé dans un grand nombre de protéines, regroupées dans une superfamille dont le prototype est la protéine 4.1 [28]. Cette superfamille contient également la merline/schwannomine, le produit $d^{\prime}$ un gène suppresseur de tumeurs responsable de la neurofibromatose de type II.

Dans les cellules en culture, les ERM sont présentes dans des structures dynamiques de la membrane riches en actine telles que les microvillosités, les filopodes et lamellipodes, tandis que dans les organes, elles présentent une spécificité tissulaire d'expression, l'ezrine et la moésine étant présentes respectivement au pôle apical des cellules épithéliales et endothéliales [29].

Sur la base de l'homologie des ERM avec la protéine 4.1, une fonction de liaison des filaments d'actine à la membrane a été proposée pour les ERM. Cependant, il a fallu attendre plus de dix ans avant que les premières informations sur les mécanismes moléculaires impliqués dans I'interaction des ERM avec leurs partenaires soient obtenues, et avant que des fonctions soient assignées à ces protéines.

Comment les ERM contrôlent-elles l'organisation du cortex cellulaire? Les premières informations ont été obtenues par l'inactivation de ces protéines, ou la surexpression de leurs formes tronquées. La technique de MicroCALI (chromophore-assisted laser inactivation) a montré que I'inactivation des ERM provoque la rétraction des extensions de la membrane plasmique [30]. L'utilisation d'oligonucléotides antisens des ARNm codant pour ces trois protéines conduit à la disparition de microvillosités et à des modifications de l'adhérence de cellules épithéliales [31]. La surproduction des protéines tronquées est souvent morphogène et induit des prolongements membranaires [32], alors que ces effets morphogènes ne sont pas observés avec la protéine entière.

Comment expliquer cette observation? Les ERM sont constituées de deux domaines: un domaine globulaire amino-terminal qui interagit avec la membrane plasmique, et un domaine carboxy-terminal associé aux filaments d'actine [33]. Le site de liaison à l'actine filamenteuse est localisé dans les 35 derniers acides aminés du domaine carboxy-terminal [34]. Deux autres sites potentiels de liaison à l'actine $G$ et $F$, respectivement, ont été récemment localisés dans la partie amino-terminale des ERM [35].

L'interaction avec les protéines membranaires peut être directe: c'est le cas de I'association des ERM avec les protéines membranaires intégrales impliquées dans l'adhérence cellulaire telles que CD44, CD43 et les ICAM (intercellular adhesion molecule). En revanche, I'interaction des ERM avec des protéines membranaires tels que le CFTR (cystic fibrosis transmembrane conductance regulator) ou l'échangeur $\mathrm{Na}^{+} / \mathrm{H}^{+}$est indirecte et relayée par des phosphoprotéines à domaines $\mathrm{PDZ}$, les protéines NHE-RF/EBP50.

L'identification des partenaires des ERMs a révélé des propriétés importantes de ces protéines. Elles sont capables de former des interactions intra- et intermoléculaires via leurs domaines amino- et carboxy-terminaux, N-ERMAD et C-ERMAD (ezrin radixin moesin association domains). Ces interactions masquent les sites d'interactions des ERM avec leurs partenaires. Par exemple, NHERF/EBP50 est incapable de lier I'ezrine sauvage, alors que l'inactivation du domaine C-ERMAD permet cette association [36]. L'association de ces protéines avec la membrane et le cytosquelette d'actine devrait donc être réglée par des changements de conformation de ces protéines, et cette hypothèse est confirmée par différentes observations. Les ERM existent dans le cytoplasme sous une forme inactive, et l'activation des ERM conduit à I'oligomérisation de ces protéines, à leur recrutement à la membrane et à leur interaction avec les filaments d'actine. Cependant, ces différentes étapes n'ont pas été formellement démontrées, et des expériences de reconstitution in vitro devraient permettre $d$ 'analyser les séquences intervenant dans I'activation des ERM (figure 6).

L'identification des signaux permettant l'activation des ERM demeure un point majeur à élucider afin de comprendre leur mode d'action. Les ERM étant les substrats de diverses tyrosine et thréonine/sérine kinases, la phosphorylation pourrait être un moyen d'activer ces protéines. En effet, dans plusieurs cas, une corrélation entre le niveau de phosphorylation des ERM et leur association avec des structures membranaires formées en réponse à des stimuli a été observée. Inversement, la déphosphorylation des protéines conduit à la dissociation des ERM de la membrane plasmique [37]. La phosphorylation d'un résidu thréonine localisé dans le site de liaison à I'actine $F$ semble être directement impliquée dans l'interaction des ERM avec I'actine $F$ in vivo aussi bien qu'in vitro [38]. De plus, la phosphorylation de cette thréonine empêche I'interaction du N-ERMAD avec le CERMAD, suggérant que cette phosphorylation maintient les ERM dans une conformation active.

La phosphorylation des ERM sur des résidus tyrosine est également nécessaire pour l'activité des ERM. Des cellules rénales cultivées dans une matrice de collagène de type I en présence d'HGF (hepatocyte growth factor) forment des structures rappelant des tubules rénaux. Cette tubulogenèse dépend de la phosphorylation de l'ezrine sur les tyrosine 145 et 353, puisque la substitution de ces résidus par des phénylalanines inhibe complètement la morphogenèse en réponse à I'HGF [39]. Enfin, les phosphoinositides pourraient avoir un rôle dans I'activation des ERM puisque, in vitro, le PiP2 augmente l'affinité des ERM pour les protéines membranaires et l'actine.

Plusieurs observations suggèrent qu'il existe un lien étroit entre les ERM et la voie de signalisation relayée par la petite GTPase Rho. Dans des cellules transfectées avec une forme active de RhoA (V14RhoA), les ERM phosphorylées sur la thréonine conservée du domaine carboxy-terminal sont recrutées dans les microvillosités induites par RhoA. In vitro, I'interaction des ERM avec RhoGDI lève l'inhibition de Rho exercée par RhoGDI et permet son activation par le facteur d'échange Dbl. L'activation des ERM est requise pour cette interaction [40]. Mais les ERM interviennent également en aval de l'activation de Rho car elles sont nécessaires à la formation des cables d'actine et des points focaux dépendant de Rho [41]. II 


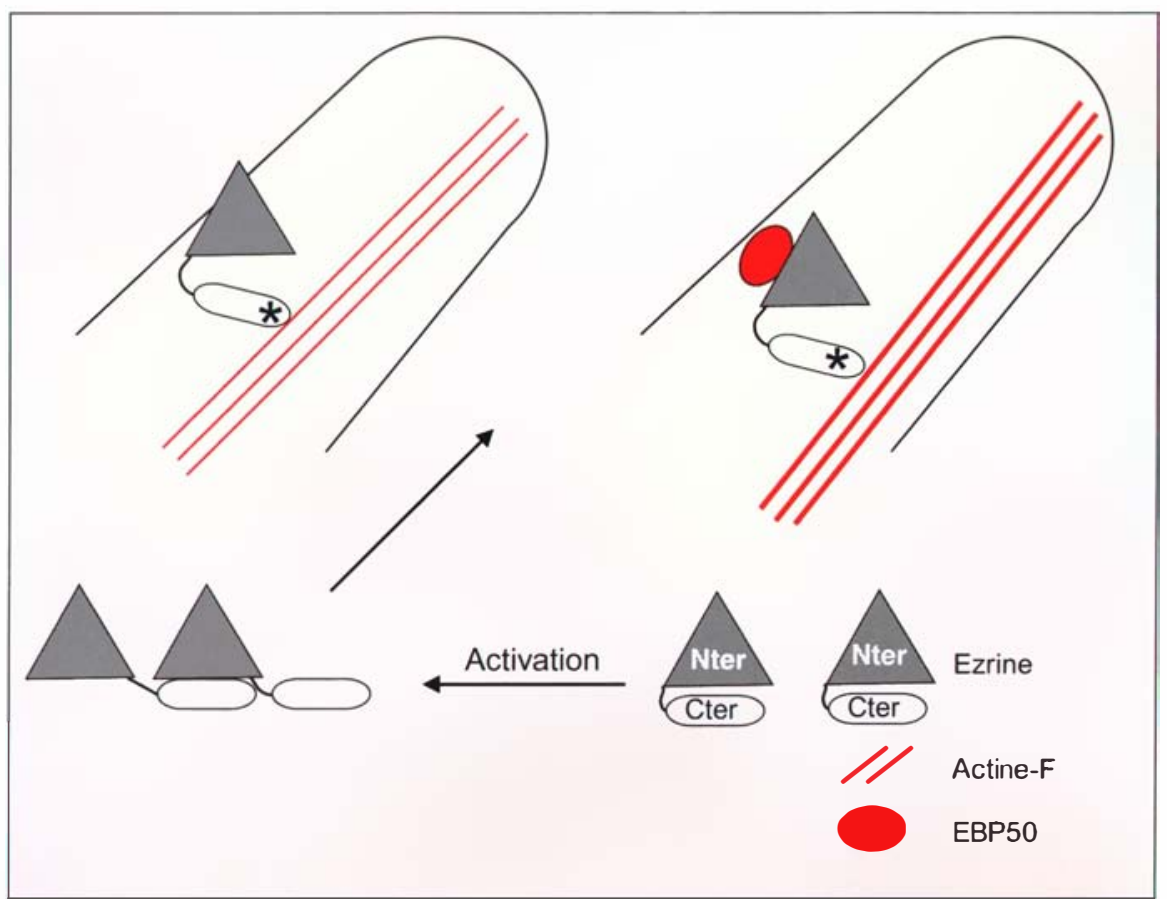

Figure 6. Modèle d'activation de l'ezrine. Dans le cytoplasme, les monomères d'ezrine sont dans une conformation inactive, en raison d'une interaction intramoléculaire entre le domaine amino-terminal et le domaine carboxy-terminal. L'ouverture conformationnelle permettrait de démasquer les sites cryptiques de liaison aux protéines membranaires et aux filaments $d^{\prime}$ actine. La liaison aux protéines membranaires peut être directe ou relayée par une phosphoprotéine, NHERF/EBP50. Les signaux qui conduisent à l'activation de l'ezrine ne sont pas connus.

semble donc exister une boucle de régulation entre la GTPase Rho et les ERM. Cependant, I'activation des ERM par un effecteur de Rho n'a pas été mise en évidence.

Les travaux effectués au cours de ces cinq dernières années ont montré que les ERM interagissent avec divers partenaires membranaires et différentes voies de signalisation. Peu d'informations sur le rôle des ERM dans le contrôle de l'organisation des filaments d'actine ont été obtenues à ce jour. En revanche, l'implication des ERM dans diverses fonctions cellulaires a déjà été proposée. Ainsi, les ERM liées aux filaments d'actine pourraient régler l'activité de protéines transmembranaires tels que le CFTR ou l'échangeur $\mathrm{Na}^{+} / \mathrm{H}^{+}$, en recrutant des protéines de régulation $[42,43]$, ou tels que le récepteur $\beta 2$ adrénergique en réglant son endocytose [44].

Un rôle direct de l'ezrine dans la transmission de signaux de survie des cellules épithéliales a récemment été établi. Des cellules produisant de processus cellulaires qui en découlent de manière indépendante. Bien que nous ayons décrit l'ezrine, le complexe Arp2/3, la zyxine et les systèmes qu'ils représentent comme des entités isolées, ces protéines jouent également un rôle important dans la transmission de signaux et dans la production d'actine-F. La complexité est l'une des caractéristiques communes aux protéines qui règlent le cytosquelette d'actine et le mouvement cellulaire. Le complexe Arp2/3 est composé de 7 protéines dont 5 ont une fonction inconnue, et la zyxine et l'ezrine forment des complexes multiprotéiques avec un nombre indéterminé de partenaires. II faut ajouter à cette complexité structurale une dimension spatiale, que I'on peut observer dans différents compartiments d'une cellule. Ainsi, la compréhension au niveau moléculaire de la manière dont le cytosquelette d'actine contrôle le mouvement cellulaire peut sembler une tâche impossible. Cependant, I'observation d'un organisme en développement, de la formation de tissus, ou de la réparation de blessures, montre que ces mouvements sont parfaitement contrôlés. Peut-être est-ce justement l'élégance du mouvement cellulaire qui nous pousse à comprendre la machinerie qui le sous-tend été muté en phénylalanine ne sont plus capables de former des tubules dans une matrice de collagène et entrent en apoptose. Le mécanisme par lequel l'ezrine transmet un signal de survie a été identifié. L'interaction de la tyrosine 353 phosphorylée avec le domaine $\mathrm{C}-\mathrm{SH} 2$ de la Pl3-kinase conduit à l'activation de la $\mathrm{PI} 3-\mathrm{K}$ et donc d'Akt (m/s 1999, $n^{\circ}$ 6/7, p. 897), une kinase nécessaire à la survie des cellules. L'apoptose observée dans les cellules exprimant l'ezrine mutée est donc due à un défaut d'activation de la voie PI3-K/Akt [45]. Ainsi, cette expérience fournit un exemple de la régulation de la survie des cellules par une protéine liant l'actine.

L'ezrine est un exemple de protéine capable d'intégrer des signaux qui contrôlent la dynamique de I'actine- $F$ et des événements majeurs tels que la motilité ou la survie. En raison des problèmes inhérents à l'étude de systèmes dynamiques, il est parfois nécessaire d'étudier les mécanismes de polymérisation de l'actine, et les

\section{Remerciements}

Nous remercions tous nos collègues de I' "Unité de morphogenèse et signalisation cellulaires" pour leurs discussions et commentaires sur ce travail. Nous regrettons de n'avoir pas cité de nombreux travaux de nos collègues, en raison des normes éditoriales. Ce travail a été financé par l'ARC (Contrats n 1312 . 9622).

\section{RÉFÉRENCES}

1. Theriot JA, Mitchison TJ. Actin microfila ment dynamics in locomoting cells. Nature $1991 ; 352: 126-31$.

2. Machesky LM, Insall RH. Signaling to actin dynamics. J Cell Biol 1999; 146: 267-72.

3. Welch MD, DePace AH, Verma S, Iwamatsu A, Mitchison TJ. The human Árp2/3 complex is composed of evolutionarily conserved subunits and is localized to cellular regions of dynamic actin filament assembly. JCell Biol 1997; 138: 375-84.

4. Welch MD, Rosenblatt J, Skoble J, Portnoy DA, Mitchison TJ. Interaction of human Arp 2/3 complex and the Listeria monocytogenes ActA protein in actin filament nucleation. Science 1998; 281: 105-8. 


\section{RÉFÉRENCES}

5. Cossart $P$, Lecuit $M$. Interactions of Listeria monocytogenes with mammalian cells during entry and actin-based movement: bacterial factors, cellular ligands and signaling. $E M B O J 1998 ; 17: 3797-806$.

6. Tilney LG, Portnoy DA. Actin filaments and the growth, movement, and spread of the intracellular bacterial parasite, Listeria monocytogenes. / Cell Biol 1989; 109: 1597-608.

7. Loisel TP, Boujemaa R, Pantaloni D, Carlier MF. Reconstitution of actin-based motility of Listeria and Shigella using pure proteins. Nature 1999; 401: 613-6.

8. Mullins RD Pollard TD. Structure and function of the Arp2/3 complex. Curr Opin Struct Biol 1999; 9: 244-9.

9. Svitkina TM, Borisy GG. Arp2/3 complex and actin depolymerizing factor/cofilin in dendritic organization and treadmilling of actin filament array in lamellipodia. $J \mathrm{Cell}$ Biol 1999; 145: 1009-26.

10. Rohatgi R, Ma L, Miki H, Lopez M, Kirchausen $H$, Takenawa $T$, Kirschner $M$. The interaction between N-Wasp and the Arp2/3 complex links Cdc42-dependent signals to actin assembly. Cell 1999; $97: 221-31$.

11. Symons $M$, Derry (M), Karlak B, et al. Wiskott-Aldrich Syndrome Protein, a novel effector for the GTPase CDC42Hs, is implicated in actin polymerization. Cell 1996 $84: 723-34$.

12. Yarar D To W, Abo A, Welch MD. The Wiskott-Aldrich syndrome protein directs actin-based motility by stimulating actin nucleation with the Arp2/3 complex. Cur Biol 1999; 9: 555-8.

13. Zalcman $\mathrm{G}$, Closson V, Honoré N, Olofsson B, Tavitian A. Participation de la cascade des gènes $R$ ho à la régulation du cytosquelette: rôle possible dans les mécanismes d'oncogénèse. Med Sci 1995; 11; 1551-6.

14. Kimura $K$, Ito $M$, Amano $M$, et al. Regulation of myosin phosphatase by Rho and Rho-associated kinase (Rho-kinase). Science $1996 ; 273: 245-8$

15. Chrzanowska-Wodnicka $M$, Burridge $K$. Rho-stimulated contractility drives the formation of stress fibers and focal adhesions. Cell Biol 1996; 133: 1403-15.

16. DePasquale J, Izzard C. Evidence for an actin-containing cytoplasmic precursor of the focal contact and the timing of incorporation of vinculin at the focal contact. / Cell Biol 1987; 105: 2803-9.

17. Hotchin N, Hall A. The assembly of integrin adhesion complexes requires both extracellular matrix and intracellular rho/rac GTPases. J Cell Biol 1995; 131 : 1857-65.

18. Beckerle $M C$. Zyxin: zinc fingers at sites of cell adhesion. BioEssays 1997; 19: 949-57.

19. Golsteyn RM, Beckerle MC, Koay T, Friederich E. Structural and functional similarities between the human cytoskeletal protein zyxin and the ActA protein of Listeria monocytogenes. J Cell Sci 1997; 110 1893-906.

20. Niebuhr $K$, Ebel $F$ Frank $R$, et al. A novel proline-rich motif present in ActA of Listeria monocytogenes and cytoskeletal proteins is the ligand for the EVH1 domain, a protein module present in the EnaNASP family. $E M B O$ J 1997; 16 : 5433-44.

21. Petit MR, Fradelizi J, Golsteyn RM, et al. LPP an actin cytoskeleton protein related to zyxin, harbours a nuclear export signal and transcriptional activation capacity. Mol Biol Cell 2000; 11:117-29.

22. Gertler FB, Niebuhr $K$, Reinhard $M$ Wehland I Soriano $P$. Mena a relative of VASP and Drosophila enabled, is implicated in the control of microfilament dynamics. Cell 1996; 87: 227-39.

23. Reinhard M, Giehl C, Abel K, et al. The proline-rich focal adhesion and microfilament protein VASP is a ligand for profilins. $E M B O$ / 1995; 14 : 1583-9.

24. Chakraborty $T$, Ebel $F$, Domann $E$, et al. A focal adhesion factor directly linking intracellularly motile Listeria monocytogenes and Listeria ivanovii to the actin-based cytoskeleton of mammalian cells. EMBO $1995 ; 14: 1314-21$.

25. Lanier LM, Gates MA, Witke W, et al. Mena is required for neurulation and commissure formation. Neuron 1999; 22:313-25.

26. Rottner K, Behrendt B, Small JV Wehland I. VASP' dynamics during lamellipodia protrusion. Nat Cell Biol 1999; 1: 321-2.

27. Bretscher A. Purification of an 80,000 dalton protein that is a component of the isolated microvillus cytoskeleton, and its ocalization in nonmuscle cells. / Cell Bio $1983 ; 97: 425-32$

28. Chishti AH, Kim AC, Marfatia SM, et al. The FERM domain: a unique module involved in the linkage of cytoplasmic proteins to the membrane. Trends Biochem Sci 1998 . $23: 281-2$.

29. Berryman $M$, Franck Z, Bretscher A Ezrin is concentrated in the apical microvilli of a wide variety of epithelial cells whereas moesin is found primarily in endothelial cells. J Cell SCi 1993; 105: 1025-43.

30. Lamb RF, Ozanne BW, Roy C et al. Essential functions of ezrin in maintenance of cell shape and lamellipodial extension in normal and transformed fibroblasts. Curr Biol 1997; 7: 682-8.

31. Takeuchi K, Sato N, Kasahara $H$, et al. Perturbation of cell adhesion and microvilli formation by antisense oligonucleotides to ERM family members. I Cell Biol 1994 . 125: 1371-84.

32. Martin $M$, Roy $P$, Montcourrier $P$, Sahuquet $A$, Mangeat $P$.' Three determinants in ezrin are responsible for cell extension activity. Mol Biol Cell 1997; 8: 1543-57.

33. Algrain $M$, Turunen $O$, Vaheri $A$, Louvard D, Arpin M. Ezrin contains cytoskeleton and membrane binding domains accounting for its proposed role as a mem- brane-cytoskeletal linker. J Cell Biol 1993; 120: 129-39.

34. Turunen $\mathrm{O}$, Wahlström $\mathrm{T}$, Vaheri $\mathrm{A}$. Ezrin has a $\mathrm{COOH}$-terminal actin-binding site that is conserved in the ezrin protein family. J Cell Biol 1994; 126: 1445-53.

35. Roy C, Martin M, Mangeat P. A dual involvement of the amino-terminal domain of ezrin in F- and G-actin binding. I Biol Chem 1997; 272 : 20088-95.

36. Reczek D, Bretscher A. The carboxy-terminal region of EBP50 binds to a site in the amino-terminal domain of ezrin that is masked in the dormant molecule. I Biol Chem $1998 ; 273: 18452-8$.

37. Chen J, Cohn JA, Mandel LJ. Dephosphorylation of ezrin as an early event in renal microvillar breakdown and anoxic injury. Proc Natl Acad Sci USA 1995; 92 : 7495-9.

38. Nakamura F, Huang L, Pestonjamasp K Luna EJ, Furthmayr $\mathrm{H}$. Regulation of F-actin binding to platelet moesin in vitro by both phosphorylation of threonine 558 and polyphosphoinositides. Mol Biol Cell 1999; 10: 2669-85.

39. Crepaldi T, Gautreau A, Comoglio PM Louvard D Arpin M. Ezrin is an effector of HGF-mediated migration and morphogenesis in epithelial cells. J Cell Biol 1997;138: 423-34.

40. Takahashi K, Sasaki T, Mammoto A, et al. Direct interaction of the Rho GDP dissociation inhibitor with ezrin/radixin/moesin initiates the activation of the Rho small $C$ protein. J Biol Chem 1997; 272: 23371-5.

41. Mackay DJG, Esch F, Furthmayr $\mathrm{H}$, Hall $A$. Rho- and Rac-dependent assembly of focal adhesion complexes and actin filaments in permeabilized fibroblasts: an essential role for ezrin/radixin/moesin proteins. I Cell Biol 1997; 138: 927-38.

42. Lamprecht G Weinman El, Yun CHC The role of NHERF and E3KARP in the CAMP-mediated inhibition of NHE3. J Biol Chem 1998; $273: 29972-8$.

43. Short DB, Trotter KW, Reczek D, et al. An apical PDZ domain anchors the cystic fibrosis transmembrane conductance regulator to the cytoskeleton. I Biol Chem 1998 . 273: 19797-801.

44. Cao TT, Deacon HW, Reczek D, Bretscher A von Zastrow M. A kinase-regulated PDZ-domain interaction controls endocytic sorting of the $\beta 2$-adrenergic receptor. Nature $1999 ; 401: 286-90$

45. Gautreau A, Poullet P, Louvard D, Arpin $M$ Ezrin a plasma membrane-microfilament linker, signals adhesion-dependent survival in epithelial cells. Proc Natl Acad Sci USA 1999; $96: 7300-5$.

\section{TIRÉS À PART}

D. Louvard. 


\section{ms 2000 \\ Summary}

The proteins of the actin cytoskeleton: well placed for motility

The cells of our body are capable of a tremendous variety of movements that require the coordination of the actin cytoskeleton, a complex network of proteins. At the heart of this network, actin is attributed with the dynamic properties of alternating between a monomer and a long polymer chain. One of the major problems in biology has been to understand how the simple process of actin polymerisation and depolymerisation can orchestrate the complex cell movement necessary for embryogenesis, immune response, and wound repair. In this review, we focus on three different, but related systems that play a major role in regulating the actin cytoskeleton. Two of these systems, represented by the Arp2/3 complex and zyxin, appear to be important in controlling actin polymerisation by different mechanisms acting at different locations in the cell. A third major system is represented by ezrin, which, in addition to its direct interaction with actin, can regulate the actin cytoskeleton and affect cell movement and survival. To unravel the complexities of cell movement and the actin cytoskeleton has been a major challenge, but the use of many different experimental systems has now given us an integrated view of cell motility.

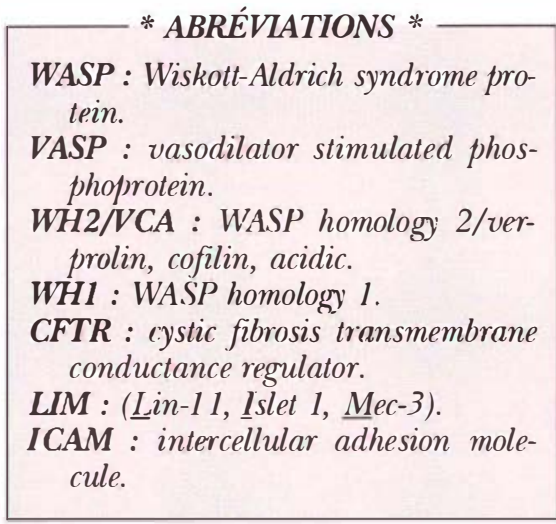

\section{APPEL D'OFFRES}

\section{Fondation Bayer Santé}

Attribution de bourses à « une équipe hospitalière »

Pour que les équipes médicales exerçant dans un service hospitalier ou une structure extra-hospitalière puissent bénéficier d'un soutien financier, la Fondation Bayer Santé a décidé de distribuer trois bourses d'un montant annuel de $100000 \mathrm{~F}$ chacunes (deux bourses à une équipe hospitalière et une bourse à une équipe para-médicale) pour soutenir un projet original visant à améliorer :

- l'accès aux soins

- la prise en charge de malades atteints de pathologies chroniques

\section{Peuvent postuler :}

- Tous les médecins et professionnels de santé membres d'une équipe n'appartenant pas ou ne travaillant pas dans un CHU ou CHR.

- Le médecins ou chercheurs n'appartenant pas à un organisme de recherche comme l'INSERM ou le CNRS.

Les équipes intéressées doivent faire parvenir à la Fondation Bayer Santé en 10 exemplaires :

- Le curriculum vitae du chef du projet.

- Un résumé du projet en 3 pages dactylographiées (double interligne) maximum. Celui-ci peut concerner une seule équipe ou associer 2 ou 3 équipes de disciplines identiques ou complémentaires. Ce résumé doit comporter: Prérequis - Hypothèse de travail Population étudiée - Méthodes - Résultats attendus - Faisabilité Calendrier - Modalités d'évaluation.

- Une justification des dépenses engagées.

Les projets soumis seront examinés par les membres du Conseil d'Administration.

Une évaluation du travail réalisé sera effectuée par le Conseil d'Administration de la Fondation Bayer Santé, 12 mois après l'attribution effective de la bourse de recherche.

L'excellence d'un travail en cours, mais non terminé, peut justifier le renouvellement de la bourse pur une deuxième année.

\section{Date limite de réception des demandes de subvention : 30 novembre 2000}

Pour tout renseignement complémentaire et envoi des dossiers candidats, s'adresser par écrit à :

Fondation Bayer Santé - Bourses Hospitalières Secrétariat de coordination :

Caroline Butelet

PR International : BP 201575761 Paris cedex 16

Contact Presse : PR International

Magalie Alphonse/Catherine Teytot Tél. : 0144058314 ou 15 